\title{
A Comparison of Stochastic Claims Reserving Methods
}

\author{
Sukriye Tuysuz and Pervin Pekel
}

\begin{abstract}
In order to preserve their solvency, it is very important for insurance companies to accurately estimate their future required reserves. The aim of this article is to determine reserves by using different stochastic models: 1) distributionfree model (Mack's model), 2) probability distribution based models (Normal, Poisson, Gamma and Inverse Gaussian distributions), and 3) these latter probability based models combined with bootstrapping. To implement these models we used data on life insurance and non-life insurance. Our findings indicate among distribution based methods, Mack's model (dataset 1 and 2) and Gamma probability distribution based model (dataset 3) are the best model in estimating reserves. The model based on Normal distribution produces the worst results, whatever the dataset. Regarding results of bootstrapping based on probability distribution models, they show that method based on Normal probability distribution (dataset 1 and 3) and ODP distribution (dataset 2) fit better. Our results also indicate that bootstrap method based on Chain-Ladder performs quit similarly than the best fitting probability distribution based bootstrap models. Among all retained models, methods based on bootstrapping present higher good-of-fit.
\end{abstract}

Index Terms - Technical Provision, Chain Ladder, Stochastic Models, Bootstrapping.

\section{INTRODUCTION}

Occurred claims of policyholders, in a given year, are usually reported with delay to the insurance company. Due to this delay, the payout is delayed too. To accomplish their obligations, insurance companies have to hold reserves. In order to preserve their solvency, it is very important for insurance companies to predict accurately their reserves. ${ }^{1}$ These companies should also take into account the risk of insufficient of the estimated reserves. The insufficient risk is then the risk of a negative run-off result, due mainly to unexpectedly large claims payments or due to the change of reported claims trend. The aim of this paper is to estimate/predict future reserves as well as the uncertainty related to these estimated values.

Initially reserves have been determined with deterministic models (Chain-Ladder, BF, Cap-Cod,...). These models are still frequently used due to their simplicity. However, they produce a point estimate of future reserves and then do not enable to formalize uncertainty related to these estimates. Stochastic models enable to remedy this drawback by determining a distribution of future reserves.

Distributions of future reserves can be obtained by using either methods based on moments, or methods based on probability distribution, or methods based on bootstrapping or hybrid methods combining those methods. Initial method based on moments, enabling to determine the error of prediction, was proposed by [1]. Another type of distribution-

S. Tuysuz is with Yeditepe University, International Finans Department, Istanbul-TURKEY (e-mail: skr.tuysuz@gmail.com).

${ }^{1}$ Reserves represent the outstanding and the Incurred But Not Enough Reported (IBNER) and the Incurred But Not Reported (IBNR) in case used observed data are claims amounts or paid claims. Predicted reserves, by using free approach is bootstrapping. These methods give a full predictive distribution without further assumptions. Methods based on probability distribution were also proposed to determine the distribution of reserves. Initially, the lognormal model was introduced by [2]. These methods based on probability distribution have been extended by several authors ([3]; [4]; [5]; [6]; [7]; [8]). Most of these extensions consisted of retaining different probability distributions (Gamma, Over-Dispersed-Poisson, Inverse Gaussian, Negative Binomial probability distributions). The estimation of these models was mainly done by using a generalized linear model. Further, these latter stochastic models were extended by combining the probability distribution based models with bootstrapping. This approach was proposed initially by [9].

The aim of this article is to determine future reserves by using different stochastic models: 1) Mack's model (distribution free model), 2) probability distribution based models (Normal, Poisson, Gamma and Inverse Gaussian distributions), and 3) these latter probability based models combined with bootstrapping. To implement these models we used data on life-insurance and non-life insurance. Lifeinsurance dataset comprises data on the Austrian claims numbers and claims amounts used by [10] in the discussion notes. Regarding the non-life insurance, the dataset used by [11] was considered. This dataset represents paid claims on Automatic Facultative General Liability (excluding asbestos and environmental) which is part of non-life insurance.

A literature review is done in section 2. Section 3 presents the datasets and the methodologies used in this article. Results are displayed and discussed in section 4. Finally, section 5 concludes the article.

\section{Literature ReVIEW}

\section{A. Deterministic methods}

Initially future reserves were determined by using the Chain Ladder (CL) method. This method consists of determining development factors (link factors) between two development years $(\mathrm{j}-1$ and $\mathrm{j})\left(\hat{f}_{j}\right)$ by dividing the sum of cumulative claims $\left(D_{i j}\right)$ associated to development year $\mathrm{j}$ by the sum of cumulative claims of the previous development years $j-1$, such as:

$\hat{f}_{j}=\frac{\sum_{i=1}^{n-j+1} D_{i, j}}{\sum_{i=1}^{n-j+1} D_{i, j-1}}$

with $i=1, \ldots, n$ and $j=1, \ldots, n-i+1$, where $n$ is the last development year. The cumulative realized claims is determined by summing realized incremental claims $\left(C_{i, j}\right)$ as: $D_{i, j}=\sum_{k=1}^{j} C_{i, k}$.

incurred claims (paid + outstanding), represent IBNR and IBNER. For claims number, predicted values correspond to IBNR. 
By using these development factors, it is possible to determine future reserves (claims) as follows:

$$
\begin{aligned}
& \widehat{D}_{i, n-i+2}=D_{i, n-i+1} \hat{f}_{n-i+2}, \\
& \widehat{D}_{i, k}=\widehat{D}_{i, k-1} \hat{f}_{k},
\end{aligned}
$$

where $D_{i, n-i+1}$ is the last observed cumulative claims associated to the accident year $\mathrm{i}$.

For each accident year (i), reserve is equal to the sum of expected future incremental reserves (claims) over the remaining development periods $\left(\delta_{i}=n-i+2, n-i+\right.$ $3, \ldots, n)$ :reserve $_{i}=R_{i}=\sum_{j \epsilon \delta_{i}} \hat{C}_{i, j}$. The ultimate reserves for the accident year $i$ is the sum of observed incremental claims and expected incremental reserves related to the accident year $i$ : ): Ultimate $_{i}=\sum_{j=1}^{n-i+1} C_{i, j}+\sum_{j \in \delta_{i}} \hat{C}_{i, j}$. The expected cumulative reserves $\left(\widehat{D}_{i, k}\right)$ are determined as: $\widehat{D}_{i, k}=\sum_{l=n-i+2}^{n-i+k} \hat{C}_{i, l}$, where $2 \leq k \leq n$.

In this CL method, future reserves are determined by using only past observed claims. Compared to this method, Bornheutter-Fergusson (BF) enables to determine future reserves by using past observations on paid claims as well as earned premium and estimated percentage of claims not yet reported. Precisely, IBNR (reserve) is determined as follows: IBNR $=$ Earned Premium*expected Ultimate Loss ratio*estimated percentage of claims not yet reported.

The expected ultimate loss ratio is determined internally by experts based on past and actual information. Regarding the expected percentage of claims not yet reported can be found out from ultimate claims and link factors determined with the CL method.

The Cape Cod method is similar to the BF method. However, instead of being in input the ultimate loss ratio is estimated based on claims reported to date.

The presented models, known as deterministic methods, provide a point estimate of the future reserves. Uncertainty, related to model parameters and to the data, are not taken into account in these models. As stated by several authors, such as [1], reserves estimated with deterministic methods for the most recent accident years are very sensitive to variations of the observed data. Moreover, different deterministic models have been proposed to determine/estimate reserves, providing widely different forecasts. It is then necessary to compare them. Furthermore, the risk of a negative run-off result and then insufficient reserves should be considered by insurance companies. Finally, utilization of reserve distributions by insurance companies is also strongly recommended in Solvency 2. Owing to these facts, it is necessary to get a distribution of expected reserves.

Several authors have tried to improve/extend deterministic models in order to get a distribution of outcomes ( [2], [3], [5], [12], [7], [13], [14], [15], [16], [17], [1], [18], [19], [20], [21], [22], [23], [24], [9], [25].). Reserves distribution has been mainly determined by using stochastic models.

\section{B. Stochastic methods}

Variability and uncertainty related to the estimated future reserves can be due to uncertainty related to the data and parameters in reserves. This variability/uncertainty, corresponding to the prediction error, is measured by the mean squared error of prediction (MSEP), which is expressed as:

$$
\begin{aligned}
& \operatorname{MSEP}\left(\hat{C}_{i, j}\right) \approx \operatorname{Var}\left(C_{i, j}\right)+\operatorname{Var}\left(\hat{C}_{i, j}\right), \\
& \operatorname{MSEP}\left(\hat{R}_{i}\right) \approx \operatorname{MSEP}\left(\sum_{j \in \delta_{i}} \hat{C}_{i, j}\right), \\
& \operatorname{MSEP}(\hat{R}) \approx \operatorname{MSEP}\left(\sum_{i=2}^{n} \widehat{R}_{i}\right),
\end{aligned}
$$$$
\underbrace{E\left[\left(C_{i, j}-\hat{C}_{i, j}\right)^{2}\right]}_{\text {prediction variance }} \approx \underbrace{E\left[\left(C_{i, j}-E\left(C_{i, j}\right)\right)^{2}\right]}_{\text {process variance }}+\underbrace{E\left[\left(\hat{C}_{i, j}-E\left(\hat{C}_{i, j}\right)\right)^{2}\right]}_{\text {estimation variance }}
$$

where $\hat{R}$ is the estimated overall reserves.

The uncertainty in reserves can be modeled with different methods: methods based on moments, methods based on probability distribution, methods based on bootstrapping and hybrid methods combining those methods. Method based on moments (model-free methods) was initially proposed by [1] and has been further reconsidered by [26] and [27]. Another type of distribution-free approach is bootstrapping. This method gives a full predictive distribution without further assumptions. Bootstrapping can also be based on probability distribution (model-based approach). Finally, model-based (distributionbased) methods enable also to determine a distribution of possible outcomes as well as uncertainty in reserves.

1) Model-free methods: In 1993 [1] proposed a distributionfree stochastic model on claims reserving. This method is based on the CL method in the estimation of reserves and the ultimate claims. Based on the mean and the standard deviation of incremental claims $\left(\mathrm{C}_{\mathrm{ij}}\right)$, this model enables to estimate the (mean squared) prediction error (MSEP) of chain-ladder estimates of reserves $\left(\mathrm{R}_{\mathrm{i}}\right)$, as follows:

$$
\begin{gathered}
\operatorname{MSEP}\left(\hat{R}_{i}\right)=\underbrace{\hat{D}_{i, n}^{2} \sum_{j=n-i+1}^{n-1} \frac{\hat{\sigma}_{j+1}^{2}}{\hat{\hat{f}}_{j+1}^{2} \hat{D}_{i, j}}}_{\text {process variance }}+\underbrace{\hat{D}_{i, n}^{2} \sum_{j=n-i+1}^{n-1} \frac{\hat{\sigma}_{j+1}^{2}}{\hat{\hat{f}}_{j+1}^{2} \sum_{q-1}^{n-j} D_{q j}}}_{\text {estimation variance }} \\
\operatorname{MSEP}(\widehat{R})=\operatorname{MSEP}\left(\sum_{i=2}^{n} \hat{R}_{i, j}\right) \\
=\sum_{i=2}^{n}\left[\operatorname{MSEP}\left(\hat{R}_{i}\right)\right]+\hat{D}_{i, n}^{2}\left(\sum_{q=i+1}^{n} D_{q, n}\right) \sum_{j=n-i+1}^{n-1} \frac{2 \hat{\sigma}_{j+1}^{2}}{\lambda_{j+1}^{2} \sum_{q=1}^{n-1} D_{q, j}}(8)
\end{gathered}
$$

where the standard deviation of development period is given as:

$$
\hat{\sigma}_{j}^{2}=\frac{1}{n-j} \sum_{i=1}^{n-j+1}\left(f_{i, j}-\hat{f}_{j}\right)^{2} .
$$

This distribution-free approach of Mack has been reconsidered and extended by some authors; such as [21], [26], [27] and [28].

2) Probability distribution based methods: Contrary to Mack's approach, a second class of approaches consists of formalizing the dynamic of reserves with a probability distribution. Early work on stochastic models, proposed by [29], consisted of modeling the logarithm of the incremental claims amounts with a Poisson probability distribution. These authors formalize the expected values of incremental reserves (claims) $\left[\mathrm{E}\left(\mathrm{C}_{\mathrm{ij}}\right)\right]$ as follows:

$$
\begin{aligned}
& E\left(C_{i, j}\right)=x_{i} y_{j}=m_{i, j}, \\
& \begin{aligned}
\ln \left(E\left(C_{i, j}\right)\right)= & \ln \left(m_{i, j}\right)=\underbrace{\ln \left(x_{i}\right)}_{\alpha_{i}}+\underbrace{\ln \left(y_{j}\right)}_{\beta_{i}}, \\
& =\mathrm{c}+\alpha_{i}+\beta_{i}+\varepsilon_{i, j}=\omega_{i, j},
\end{aligned}
\end{aligned}
$$

where $\mathrm{x}_{\mathrm{i}}$ and $\mathrm{y}_{\mathrm{j}}$ represent the expected ultimate claims and the proportion of ultimate claims, respectively. Estimation is performed with Maximum Likelihood Method. 
By assuming that residuals in equation 10 are normally distributed $\left(\varepsilon_{i, j} \rightarrow N\left(0, \sigma^{2}\right)\right)$, [2] introduced the log-Normal model. This model has been considered and extended in several studies ([3]; [4]; [5]; [6]; [7][8]). The fitted/predicted values, in [2]'s approach, are determined as:

$\hat{C}_{i, j}=\exp \left(\widehat{w}_{i, j}+0.5 \hat{\sigma}_{i, j}\right)$

With $\hat{\sigma}_{i, j}=\operatorname{Var}\left(\widehat{w}_{i, j}\right)+\sigma^{2}$,

where $\hat{\sigma}_{i, j}$ is the prediction variance of the linear predictor determined by summing the variance of the linear predictor $\left(\operatorname{Var}\left(\widehat{w}_{i, j}\right)\right)$ with the process variance $(\sigma)$. The prediction variance of a future incremental reserves (incremental claims) ( $\left.\hat{C}_{i, j}\right)$, of reserves related to accident year $\mathrm{i}\left(\hat{R}_{i}\right)$, and to the overall reserves $(\hat{R} \quad)$ are given as:

$$
\begin{aligned}
& \operatorname{MSEP}\left(\hat{C}_{i, j}\right)=\hat{C}_{i, j}^{2} \exp \left[\left(\hat{\sigma}_{i, j}^{2}\right)-1\right] \\
& \operatorname{MSEP}\left(\hat{R}_{i}\right) \approx \sum_{j \in \delta_{i}} \operatorname{MSEP}\left(\hat{C}_{i, j}\right) \\
& +2 \sum_{j_{1} j_{2} \in \delta_{i} ; j_{2}>j_{1}}^{n} \hat{C}_{i, j 1} \hat{C}_{i, j 2}\left[\exp \left(\operatorname{Cov}\left(\widehat{w}_{i, j 1}, \widehat{w}_{i, j 2}\right)\right)-1\right] \\
& \operatorname{MSEP}(\widehat{R}) \approx \sum_{i=2}^{n} \operatorname{MSEP}\left(\hat{R}_{i}\right) \\
& +2 \sum_{i 1, i 2=2}^{n} \sum_{j 1, j 2 \in \delta_{i} ; j 2>j 1} \hat{C}_{i 1, j 1} \hat{C}_{i 2, j 2} \exp \left[\left(\operatorname{Cov}\left(\widehat{w}_{i, j 1}, \widehat{w}_{i, j 2}\right)\right)-1\right]
\end{aligned}
$$

Most of the improvement proposed to [2]'s model have consisted of retaining a different probability distribution. For instance, [30] proposed to model claims dynamic with a Gamma distribution and by using a maximum likelihood method. As for [31], they proposed to model the logged incremental claims dynamic by using an Over-Dispersed Poisson (ODP) probability distribution. Inverse Gaussian distribution was also suggested. In most of these proposed models, parameters are estimated with Generalized Linear Model (GLM).

The expected mean and variance of the incremental claims in these models can be determined as:

$$
\begin{aligned}
& E\left(C_{i ; j}\right)=x_{i} y_{j}=m_{i, j}, \\
& \operatorname{Var}\left(C_{i ; j}\right)=\phi m_{i, j}^{z}, \\
& \log \left(m_{i, j}^{2}\right)=w_{i, j}=c+\alpha_{i}+\beta_{i}
\end{aligned}
$$

where $\mathrm{z}$ is a scalar. i) If the retained probability distribution is a normal distribution then $\mathrm{z}=0$, ii) If it is a over-dispersed probability(ODP) distribution then $z=1$, iii) If it is a Gamma distribution then $z=2$, iv) If it is an inverse Gaussian distribution then $\mathrm{z}=3$ and $\mathrm{v}$ ) if it is a compound Poisson distribution then $0<\mathrm{z}<1$.

The prediction variance of these models (=process variance

+ estimation variance) are given by the following expressions:

$$
\begin{gathered}
\operatorname{MSEP}\left(\hat{C}_{i, j}\right) \approx \operatorname{Var}\left(C_{i ; j}\right)+\operatorname{Var}\left(\widehat{C}_{i, j}\right) \\
\approx \phi m_{i, j}^{z}+m_{i, j}^{z} \operatorname{Var}\left(\widehat{w}_{i, j}\right), \\
\operatorname{MSEP}\left(\hat{R}_{i}\right)=\operatorname{MSEP}\left(\sum_{j \in \delta_{i}} \widehat{C}_{i, j}\right), \\
\approx \sum_{j \in \delta_{i}} \phi \widehat{m}_{i, j}^{z}+\sum_{j \in \delta_{i}} \widehat{m}_{i, j}^{z} \operatorname{Var}\left(\widehat{w}_{i, j}\right) \\
+2 \sum_{j_{1} j_{2} \in \delta_{i}, j_{2}>j_{1}} \widehat{m}_{i, j 1} \widehat{m}_{i, j 2} \operatorname{Cov}\left(\widehat{w}_{i, j 1}, \widehat{w}_{i, j 2}\right)
\end{gathered}
$$

$$
\begin{aligned}
& \operatorname{MSEP}(\hat{R})=\operatorname{MSEP}\left(\sum_{i=1}^{n} \hat{R}_{i}\right), \\
& \approx \sum_{i, j \in \delta} \phi \hat{m}_{i, j}^{z}+\sum_{i, j \in \delta} \hat{m}_{i, j}^{z} \operatorname{Var}\left(\widehat{w}_{i, j}\right) \\
& +2 \sum_{i_{1} i_{2} j_{1} j_{2} \in \delta, i_{1} j_{1} \neq i_{2} j_{2}} \widehat{m}_{i, j 1} \widehat{m}_{i, j 2} \operatorname{Cov}\left(\widehat{w}_{i, j 1}, \widehat{w}_{i, j 2}\right)
\end{aligned}
$$

Apart from the cited probability distributions, [17] derived the over-dispersed negative binomial probability distribution from the Poisson distribution. Under this distribution, the expected incremental reserves (claims) and the variance of those reserves are determined as follows:

$$
\begin{aligned}
E\left(C_{i, j}\right) & =m_{i, j}=\left(\lambda_{j-1}\right) D_{i, j-1}, \\
\operatorname{Var}\left(C_{i, j}\right) & =\Phi \lambda_{j} \lambda_{j-1} D_{i, j-1}, \\
\log \left(m_{i, j}\right) & =\log \left(\lambda_{j}-1\right)+\log \left(D_{i, j-1}\right), \\
& =c+\alpha_{j-1}+\log \left(D_{i, j-1}\right)
\end{aligned}
$$

where $D_{i ; j}$ represents the cumulative incremental claims in accident year $i$ till the development year $j$. As in the CL model, $\lambda_{j}$ represents the development factor at the $\mathrm{j}$ development period. As for $\Phi$, it is the dispersion parameter.

Based on process variance and estimation variance, the overall reserve estimation variance is determined as follows ([11]):

$$
\begin{aligned}
\operatorname{MSEP}\left(\widehat{D}_{i, n}\right) \approx & \Phi D_{i, n-i+1} \prod_{k=n-i+2}^{n} \hat{\lambda}_{k}\left(\prod_{k=n+2}^{n}\left(\hat{\lambda}_{k}-1\right)\right) \\
& +\widehat{D}_{i, n-i+1}^{2} \operatorname{Var}\left(\prod_{k=n-i+2}^{n} \hat{\lambda}_{k}\right)
\end{aligned}
$$

$$
\operatorname{Var}(R) \approx \sum_{i=2}^{n} \operatorname{Var}\left(D_{i, n}\right)+2 \sum_{i=2, j>i}^{n} \operatorname{Cov}\left(\widehat{D}_{i, n}, \widehat{D}_{j, n}\right)
$$

[11] derived the negative binomial model from the Poisson model by assuming that they are closely related, but with a different parametrization. Furthermore, these authors considered the Normal model as an approximation to the negative binomial model. Without referring to the negative binomial model, [20] considered a normal linear regression. This latter approach was extended by [22].

\section{Bootstrapping methods}

Bootstrapping methods enable to generate a distribution of possible reserves (claims) instead of a point estimate. Some authors combined the bootstrap concept with a classical chain ladder model: (model-free bootstrapping) ([34],[9],[11], [32], [33]).

The bootstrapping method consists of sampling with replacement from the residuals determined by using observed incremental claims $\left(\mathrm{C}_{\mathrm{i} ; \mathrm{j}}\right)$ and fitted incremental claims $\left(\mathrm{m}_{\mathrm{i} ; \mathrm{j}}\right)$. The unscaled Pearson residuals $\left(\mathrm{r}_{\mathrm{ij}}\right)$ and scale parameter $(\Phi)$ are determined as follows:

$$
r_{i, j}=\frac{C_{i, j}-m_{i, j}}{\sqrt{m_{i, j}^{z}}} \text { and } \Phi=\frac{\sum r_{i, j}^{2}}{N-p}
$$

where $\mathrm{N}$ is the total number of observation and $\mathrm{p}$ represents the number estimated parameters.

This sampling method does not require that residuals are normally distributed but assumes that they are independent and identically distributed (i.i.d.). The Pearson residuals should be adjusted in order to obtain equal variance and unbiased residuals. This adjustment can be done by 
multiplying the Pearson residuals with a degree of freedom adjustment factors $\left(f^{D o F}=\sqrt{\frac{N}{N-p}}\right)([9] ;[34])$. The resulting scaled Pearson residuals $\left(r_{i, j}^{s}\right)$ are then just equal to: $r_{i, j}^{s}=$ $r_{i, j} f^{D o F}$. This adjustment approach was criticized by [35][32]; according to whom the obtained scaled Pearson residuals are not standardized and then do not have the same variance. These authors proposed to standardize Pearson residuals by adjusting Pearson residuals $\left(r_{i, j}\right)$ with hat adjustment factor $\left(f_{i, j}^{H}\right)$, which is determined as:

$$
f_{i, j}^{H}=\sqrt{\frac{1}{1-H_{i, j}}},
$$$$
\text { with } H_{i, j}=X\left(X^{T} W X\right)^{-1} X^{T} W \text {, }
$$

where $\mathrm{X}$ and $\mathrm{W}$ are the design and the weight matrices, respectively. The standardized residuals are then obtained by multiplying the unscaled residuals with the hat adjustment factor as: $r_{i, j}^{H}=r_{i, j} f^{H}$.

Bootstrapping by sampling with replacement consists of recreating an upper triangle of incremental claims (determining fitted claims $-\hat{C}_{i, j}$ ) as follow:

$$
\hat{C}_{i, j}=r_{i, j}^{k} \sqrt{m_{i, j}^{z}}+m_{i, j}
$$

where $r_{i, j}^{k}=r_{i, j}$ if unscaled residuals, $r_{i, j}^{k}=r_{i, j}^{s}$ if scaled residuals and $r_{i, j}^{k}=r_{i, j}^{H}$ if standardized residuals. The link ratios or model parameters are then redetermined by using these fitted values and then estimate future values (point estimates). By repeating this process several times (sample from residuals to recreate past observed upper triangle, refit past observed values, redetermine link-ratios or model parameters and then forecast future claims/reserves) a distribution of point estimates can be obtained. This approach was considered by [9], who used link-ratios. These authors did not integrate over-dispersion of residuals and process variance in their calculations but recommend to take them into account.

\section{DATA AND METHODOLOGIES}

The purpose of this paper is to determine and compare reserves by using different deterministic and stochastic models. For this analysis, three datasets are considered: the fictitious dataset, used by [10], on the number and amount of claims of Austrian life insurance. Dataset used by [11] is also retained in this article. This latter dataset represents data on Automatic Facultative General Liability (excluding asbestos and environmental) which is part of non-life insurance. Reserves predicted for the first dataset, composed with claims amounts of life insurance, represent outstanding claims and IBNER (Incurred But Not Enough Reported) and IBNR (Incurred But Not Reported). Regarding the second dataset (number of claims of life insurance), the predicted reserves correspond to the IBNR. Finally, predicted values (reserves) from the third dataset (paid claims) also represent the IBNR. Regarding the models/methods, the following are used:

- Model-free stochastic method: Mack's approach

- Model-based stochastic methods using Over-DispersedPoisson (ODP), Gamma, Normal and Inverse Gaussian probability distribution and estimated with Generalized Linear Method (GLM).

- Model-based Bootstrap methods: based on ODP, Gamma, Normal and Inverse Gaussian probability distributions, respectively,
- Model-free Bootstrap: based on Chain Ladder model.

For each bootstrap method based on probability distribution, there are two possibilities in the estimation of the lower part of the triangle and then the estimated claims/reserves. This estimation can be done either by using the model based on all parameters or the restricted model based on 3 parameters, which are defined as:

$$
\begin{aligned}
& \text { Full model } \\
& \qquad \begin{array}{l}
\log \left(m_{i, j}\right) \\
\text { Restricted model }
\end{array} \\
& \qquad \log \left(m_{i, j}\right)=w_{i, j}=c+\alpha_{\text {accident }}+\beta_{\text {development }}
\end{aligned}
$$

where c, $\alpha_{\text {accident }}$ and $\beta_{\text {development }}$ represent the calender year trend, the accident year and one the development year, respectively. Precisely, for instance, estimations using the ODP bootstrap method requires the estimation of a constant, of a parameter for each accident year and a parameter for each development year. By contrast the restricted model is based on only 3 estimated parameters.

Estimations of reserves with Mack's model and with probability distribution based stochastic models are done in $\mathrm{R}$ by using the Chain Ladder package. Regarding methods based on bootstrapping, they are implemented by using an Excel based package developed by [36].

\section{EMPIRICAL RESULTS}

\section{A. Diagnostic Test of Residuals}

Bootstrapping consists of sampling with replacement (unscaled or scaled or standardized) residuals in order to simulate the upper triangle. These residuals should be identically and independently distributed (i.i.d). They do not need to be normally distributed. We have to check whether the determined residuals are i.i.d. Several metrics exist for this purpose (see [37]). The most used tests consists of analyzing the residuals graphically and by using Normality test. The graphical analysis can be done by comparing the data points with the fitted normal distribution line. Regarding the normality test, for example the Anderson-Darling test consists of testing the null hypothesis $\left(\mathrm{H}_{0}\right)$ of normality against the alternative hypothesis $\left(\mathrm{H}_{\mathrm{a}}\right)$ of non-normality.

Bootstrapping requires also that residuals per development year (per accident year, per calendar year) are homoscedastics. In case this condition is not verified, heteroscedasticity should be taken into account during sampling process or should be corrected. Taken into account of the heteroscedasticity can be done in two ways: 1) by applying a stratified approach or 2) by adjusting residuals with hetero-adjustment factor. This latter method is applied in this paper. The Excel package ([36]) provides the models having the highest $\mathrm{R}^{2}$ by efficiently grouping development periods having similar variances. Based on these groups, heteroscedasticity adjusted standardized residuals are determined.

Results of the diagnostic tests of residuals are depicted in graphs A1.1-A3.2 (Appendix). These graphs represent standardized residuals plots, of the data 1, 2 and 3, related to bootstrap method based on ODP, Gamma and Normal probability distribution with GLM method. Charts on the left side of these graphs are specific to standardized residuals without any adjustment for heteroscedasticity and charts on the right side are associated to hetero-adjusted standardized residuals. 
According to the charts on the left part in figure A1.1 residuals look i.i.d. and normally distributed at the first glance. However, some outliers are present. Furthermore, the p-values of the normality test are equal or lower than the significance level of $5 \%$, indicating the rejection of the null hypothesis of normality. Results of the hetero-adjusted standardized residuals are displayed in the right side in charts A1-1. These results show that these hetero-adjusted residuals are more normally distributed and i.i.d. than non adjusted residuals. Indeed, the p-values are higher than the significance level of $5 \%$ in case of hetero-adjustment for all retained probability distribution. For example, the p-value increases from $36 \%$ to $99 \%$, from $1 \%$ to $72 \%$, and from $50 \%$ to $91 \%$ in case of ODP distribution, Gamma distribution and Normal distribution, respectively, after hetero-adjustment. These latter results are in favor of the null hypothesis of normality of hetero-adjusted tandardized residuals. Moreover, the numbers of outliers are reduced in case of hetero-adjusted residuals compared to nonadjusted residuals. Furthermore, the $\mathrm{R}^{2}$ are higher after heteroadjustment whatever the probability distribution. For instance, the R2 increases from \%97,5 to \%99,4 in GLM Poisson, from \%93 to $\% 98,5$ in GLM Gamma, and from $\% 97,9$ to $\% 99$ in GLM Normal after hetero-adjustment. Finally, values of the Bayesian Information Criterion (BIC) decrease after heteroadjustment of standardized residuals. The lowest BIC is reached in model using ODP distribution based on heteroadjusted standardized residuals. Results on $\mathrm{R}^{2}$ and $\mathrm{BIC}$ indicate that models using hetero-adjusted standardized residuals fit better than models using non-adjusted residuals. Appendix A1-2 represents charts of standardized residuals extracted from restricted models applied on dataset 1. Charts on the left part reveal that standardized residuals look biased and heteroscedastics. Furthermore, there are several extreme (outliers) standardized residuals (normality plots). Scatter plots on the right part reveal a significant improvement after hetero-adjustment. As in non-restricted models, the pvalues are higher than the significance level of $5 \%$ after heteroadjustment compared to non-adjusted residuals. These results show that hetero-adjusted standardized residuals are i.i.d. and normally distributed. The increases of $\mathrm{R}^{2}$ and the decreases of BIC's values indicate that models based on hetero-adjusted standardized residuals fit better than models based on non hetero-adjusted standardized residuals. According to the residuals of these three models, residuals from GLM Gamma model are more random. Furthermore, this model has the lowest BIC value.

Results related to the dataset 2 are depicted in graphs A2-1 (appendix) for the full models (with all parameters) and in A2-2 for the restricted models. Similar to dataset 1, these results show the non normality of standardized residuals ( $p$ values and normality graphs). The increases of $p$-values above the significance level of 5\% indicate the acceptance of the null hypothesis of normality. According to the increases of $\mathrm{R}^{2}$ and the decreases of the BIC's values models based on heteroadjusted residuals fit better than model based on non adjusted standardized residuals. The lowest BIC value is obtained in model based on normal distribution (full model) and gamma distribution (restricted model).

Similar findings are obtained for the third dataset. Standardized residuals are not i.i.d. and are heteroscedastics whereas hetero-adjusted standardized residuals are i.i.d. and normally distributed. The number of outliers and the BIC's values are reduced after hetero-adjustment. These latter results show that models fit better to hetero-adjusted standardized residuals than to simple standardized residuals. The lowest BIC value is obtained with the ODP model in both models (full model and reduced model).

\section{B. Presentation and discussion of results}

Tables I-IV provide results obtained related to the standard error, the coefficient of variation (C.V.) and to the reserves for each model and each dataset. These reserves represent the outstanding and the Incurred But Not Enough Reported (IBNER) and the Incurred But Not Reported (IBNR) in case used dataset are claims amounts or paid claims (dataset 1 and 3 ). These reserves correspond to the IBNR in case the used data are the number of claims (dataset 2). The coefficient of variation is determined by dividing the standard error by the reserves. The standard error represents the prediction error; which is the sum of the process error and the estimation error. Results from Mack's model and probability distribution based models are displayed in Table I. Tables II and III contain outcomes of bootstrapping based on probability distribution models (full and reduced). Results related to the bootstrapping based on deterministic model (CL model) are displayed in Table IV. Comparisons of predicted reserves do not provide efficient information on models. It is better to compare models by referring to their standard errors and coefficient of variations.

\begin{tabular}{|l|rrrr|} 
Table I & Results of Stochastic models \\
\hline Data1 & Latest & Reserves & S.E & CV(IBNR) \\
\hline Mack & 108015100 & 78857471 & 21194909 & $27 \%$ \\
Poisson & 108015100 & 78857471 & 22841755 & $29 \%$ \\
Gamma & 108015100 & 77282255 & 21500048 & $28 \%$ \\
Normal & 108015100 & 80846133 & 32067766 & $40 \%$ \\
Inverse Gaussian & 108015100 & 80233357 & 25379546 & $32 \%$ \\
\hline Data2 & & & & \\
Mack & 2274 & 1501 & 358 & $24 \%$ \\
Poisson & 2274 & 1500 & 407 & $27 \%$ \\
Gamma & 2274 & 1432 & 363 & $25 \%$ \\
Normal & 2274 & 1567 & 592 & $38 \%$ \\
Inverse Gaussian & 2274 & 1397 & 392 & $28 \%$ \\
\hline Data3 & & & & \\
\hline Mack & 161086 & 52278 & 43778 & $84 \%$ \\
Poisson & 161086 & 52278 & 34121 & $65 \%$ \\
Gamma & 161086 & 53690 & 33920 & $63 \%$ \\
Normal & 161086 & 52680 & 53623 & $102 \%$ \\
Inverse Gaussian & & & & \\
\hline
\end{tabular}

According to the first part of Table I, results relative to the life insurance data (claims amount), standard error and coefficient of variation (C.V.) determined with Mack's model are the lowest; meaning that this model is more appropriated in the calculation of reserves. These lowest values mean that estimated reserves are more concentrated (less dispersed) and the expected reserves value more efficiently represents possible reserves than the other retained models. Results on standard error and C.V. related to the Gamma and Poisson distributions are not too different from the results of Mack's model. The model based on Normal distribution provides the worst results. Indeed, the standard error and C.V. of this model are the highest. Similar findings are deduced from the estimations related to the Austrian life-insurance number of claims (part 2 Table I). Regarding the third dataset (non-life insurance claim amounts), the lowest standard errors and 
C.V. are the one deduced from the Gamma and Poisson distribution. Results of these both models are very similar. Like datasets 1 and 2, the normal probability distribution based model provides the highest variability and C.V. As for Mack's model, it is in the middle.

Table II: Probability Distribution Based Models combined with Bootstrapping - Full Models

\begin{tabular}{|l|rrrr|}
\hline Data1 & Latest & Reserves & \multicolumn{1}{c}{ S.E. } & \multicolumn{1}{c}{ CV } \\
\cline { 2 - 6 } Poisson & 108015100 & 80387997 & 16971539 & $21 \%$ \\
Gamma & 108015100 & 95410133 & 625358568 & $655 \%$ \\
Normal & 108015100 & 82574138 & 14035815 & $17 \%$ \\
Inv. Gaus. & 108015100 & 62254632 & 47236543 & $76 \%$ \\
\hline Data2 & & & & \\
Poisson & 2274 & 1528 & 226 & $15 \%$ \\
Gamma & 2274 & 1479 & 241 & $16 \%$ \\
Normal & 2274 & 1591 & 275 & $17 \%$ \\
Inv. Gaus. & 2274 & 1479 & 241 & $16 \%$ \\
\hline Data3 & & & & \\
Poisson & 161086 & 38912 & 29499 & $76 \%$ \\
Gamma & - & - & & - \\
Normal & 161086 & 49411 & 32990 & $67 \%$ \\
Inv. Gaus. & 0 & 0 & 0 & $0 \%$ \\
\hline
\end{tabular}

Inv. Gaus.: inverse Gausian. CV of reserves

Table II presents the results obtained from models using bootstrapping method based on probability distributions. Results associated to the first data show that models using Normal probability distribution and ODP distributions have the lowest C.V. values. Calculated values of C.V. of the second dataset are more close to each other with the lowest value obtained in model based on ODP distribution followed by model based on Gamma and Inverse Gaussian distribution. As for the third dataset, the lowest C.V. is obtained in bootstrap method based on Normal probability distribution.

Results of bootstrapping based on restricted probability distribution based models (Table III) show than the best fitting model having the smallest C.V. is the ODP model and the worst fitting model is the model using Inverse Gaussian probability distribution for the data 1 . The comparison between the full and restricted model reveals that the best fitting full model based on Normal distribution has lower C.V. $(17 \%)$ value than the retained restricted model using ODP distribution (27\%). Similar finding is observed for the second dataset: the best fitting full ODP model dominates the best fitting restricted model. Contrary to these findings, restricted models have lower C.V. values than full models whatever the probability distribution for the third dataset.

The comparison of all retained models shows that bootstrap method based on Normal probability distribution is the best fitting model with a C.V. value of $17 \%$ followed by the bootstrap model based on deterministic CL model (C.V. = $18 \%$ ) for the first dataset. Similarly, methods based on bootstrapping have highest goodness-of-fit in the second dataset. Precisely, the models having the smallest C.V. values are bootstrap model based on ODP distribution (C.V. $=15 \%)$ and bootstrap model based on Chain Ladder (C.V. $=15 \%)$. As for the third dataset, the lowest C.V. value is obtained with the bootstrap model based on restricted ODP distribution model $(\mathrm{C} . \mathrm{V} .=35 \%)$ followed by the bootstrap model based on restricted Normal distribution model $(\mathrm{C} . \mathrm{V} .=36 \%)$ and the bootstrap model based on deterministic CL model (C.V. = $38 \%$ ).

Table III: Probability Distribution Based Models combined with Boostrapping - Restricted Models

\begin{tabular}{|l|rrrr|}
\hline \multirow{2}{*}{ Data1 } & \multicolumn{1}{c}{ Latest } & Reserves & \multicolumn{1}{c}{ S.E. } & \multicolumn{1}{c|}{ CV } \\
\cline { 2 - 5 } Poisson & 108015100 & 113781456 & 30172969 & $27 \%$ \\
Gamma & 108015100 & 110167781 & 34106049 & $31 \%$ \\
Normal & 108015100 & 114284315 & 36262822 & $32 \%$ \\
Inv. Gaus. & 108015100 & 19801129 & 50139686 & $253 \%$ \\
\hline Data2 & & & & \\
Poisson & 2274 & 2131 & 636 & $30 \%$ \\
Gamma & 2274 & 2118 & 632 & $30 \%$ \\
Normal & 2274 & 2276 & 806 & $35 \%$ \\
Inv. Gaus. & 2274 & 2118 & 632 & $30 \%$ \\
\hline Data3 & \multicolumn{5}{|r}{} & \\
Poisson & 161086 & 75981 & 26836 & $35 \%$ \\
Gamma & - & & & \\
Normal & 161086 & 84980 & 30887 & $36 \%$ \\
Inv. Gaus. & 0 & 0 & 0 & $0 \%$ \\
\hline
\end{tabular}

Inv. Gaus. Represents the inverse Gausian.

Table IV - Chaine-Ladder based Boostrapping

\begin{tabular}{|l|rrrr|}
\hline & \multicolumn{1}{|c}{ Latest } & Reserves & \multicolumn{1}{c|}{ S.E. } & \multicolumn{1}{c|}{ CV } \\
\cline { 2 - 5 } Data1 & 108015100 & 79739441 & 14576519 & $18 \%$ \\
Data2 & 2274 & 1520 & 235 & $15 \%$ \\
Data3 & 161086 & 50748 & 19262 & $38 \%$ \\
\hline
\end{tabular}

\section{CONCLUSION}

The heteroscedasticity adjusted residuals are more randomly distributed. Furthermore, this adjustment improves the models fit by increasing their $\mathrm{R}^{2}$, decreasing their BIC's values and by reducing the number of outliers.

Among distribution based methods, Mack's model is the best model in estimating reserves in the first and the second dataset. As for the third dataset, model based on Gamma probability distribution performs best. The model based on Normal distribution produces the worst results, whatever the dataset.

Results of bootstrapping based on probability distribution models show that full models fit better than reduced models for the first and second dataset. For the third dataset, bootstrap method based on restricted model suits better. Our results also reveal that method based on Normal probability distribution fit better for the first and third dataset. Regarding the second dataset, the best performing model is the bootstrap method based on ODP. The overall results related to the bootstrapping method based on probability distributions and deterministic method indicate that bootstrap method based on CL performs quit similarly than the best fitting probability distribution based model.

The comparison of the results of all retained models shows that methods based on bootstrapping present higher goodoffit. 


\section{REFERENCES}

[1] T. Mack. Distribution-free calculation of the standard error of chainladder reserve estimates. ASTIN Bulletin, 23:213-225, 1993.

[2] E. Kremer. Ibnr claims and the two way model of anova. Scandinavian Actuarial Journal, pages 47-55, 1982.

[3] G.C. Taylor and F.R. Ashe. Second moments of estimates of outstanding claims. Journal of Econometrics, 23(1):

[4] B. Zehnwirth. Interactive claims reserving forecasting system. Insureware St.Kilda Australia, 1985.

[5] A.E. Renshaw. Chain-ladder and interactive modelling (claims reserving and glim). Journal of the Institute of Actuaries, (116):559-587, 1989.

[6] S. Christofides. Regression models based on logincremental payments. Claims Reserving Manual, Institute of Actuaries, London, 2, 1990.

[7] R.J. Verrall. Bayes and empirical bayes estimation for the chain-ladder model. ASTIN Bulletin, 20(2):217-243, 1990.

[8] R. Verrall. On the estimation of reserves from loglinear models. Insurance Mathematics and Economics, 10:75-80, 1991.

[9] P. England and R. Verrall. Analytic and bootstrap estimates of prediction errors in claims reserving. Insurance: Mathematics and Economics, 25:281-293, 1999.

[10] N. Kulikov and S. Dixon. Life insurance \& wealth management practice committee. Discussion Note: IBNR, (December 2014), 2014.

[11] P.D. England and R.J. Verrall. Stochastic claims reserving in general insurance. British Actuarial Journal, 8(3): 443-518, 2002.

[12] R.J. Verrall. A state space representation of the chainladder linear model. Journal of the Institute of Actuaries, 116(7):589-610, 1989.

[13] R.J. Verrall. On the unbiased estimation of reserves from loglinear models. Insurance: Mathematics and Economics, 10(1):75-80, 1991a

[14] R.J. Verrall. Chain-ladder and maximum likelihood. Journal of the Institute of Actuaries, 118:489-499, 1991b.

[15] R.J. Verrall. A method for modelling varying run-off evolutions in claims reserving. ASTIN Bulletin, 24(2): 325-332, 1994.

[16] R.J. Verrall. Claims reserving and generalised additive models. Insurance: Mathematics and Economics, 19:31-43, 1996.

[17] R.J. Verrall. An investigation into stochastic claims reserving models and the chain-ladder technique. Insurance: Mathematics and Economics, 26:91-99, 2000.

[18] T. Mack. Which stochastic model is underlying the chainladder model? Insurance:Mathematics and Economics, (15):133-138, 1994a.

[19] T. Mack. Measuring the variability of chain-ladder reserve estimates. Casualty Actuarial Society, Spring Forum, 1994b.

[20] D.M. Murphy. Unbiased loss development factors. PCAS LXXXI, pages 154-222, 1994.

[21] K.D. Schmidt and A. Schnaus. An extension of mack's model for the chain-ladder method. ASTIN Bulletin, 26: 247-262, 1996.

[22] G. Barnett and B. Zehnwirth. Best estimates for reserves. Casualty Actuarial Society,Fall Forum., 1998.

[23] A.E. Renshaw and R.J. Verrall. A stochastic model underlying the chain-ladder technique. British Actuarial Journal, 4(4):903-923, 1998.

[24] T. Mack and G. Venter. A comparison of stochastic models that reproduce chain-ladder reserve estimates. Insurance: Mathematics and Economics, 26:101-107, 2000.

[25] P.D. England and R.J. Verrall. A flexible framework for stochastic claims reserving. Casualty Actuarial Society, 2001.

[26] M. Buchwalder, H. Bhlmann, M. Merz, and M. Wthrich. The mean square error of prediction in the chain-ladder reserving method (mack and murphy revisited). ASTIN Bulletin, 36(2):521-542, 2006.

[27] T. Mack, G. Quarg, and C. Braun. The mean square error of prediction in the chain-ladder reserving method - a comment. ASTIN Bulletin, 36(2):543-552, 2006.

[28] M. Merz and M. V. Wthrich. Paid-incurred chain claims reserving method. Insurance: Mathematics and Economics, 46(3):568-579, 2010.

[29] S. Hachemeister and Stanard. Ibnr claims count estimation with static lag functions. Spring Meeting of the Casualty Actuarial Society, 1975.

[30] T. Mack. A simple parametric model for rating automobile insurance or estimating ibnr claims reserves. ASTIN Bulletin, 21:93-109, 1991.

[31] A.E. Renshaw and R.J. Verrall. A stochastic model underlying the chain-ladder technique. ASTIN Colloquium in Cannes, pages 45-69, 1994.

[32] P.J.R. Pinheiro, J.M.A. e Silva, and M. de Lourdes Centeno. Bootstrap methodology in claim reserving. Journal of Risk and Insurance, (70):701-714, 2003.

[33] G.S. Kirschner, K. Colin, and I. Belinda. Two approaches to calculating correlated reserve indications across multiple lines of business. Variance, 1:15-38, 2008.

[34] P. England. Addendum to 'analytic and bootstrap estimates of prediction error in claims reserving'. Insurance: Mathematics and Economics, 31:461-466, 2002.
[35] P.J.R. Pinheiro, J.M.A. e Silva, and M. de Lourdes Centeno. Bootstrap methodology in claim reserving. ASTIN Colloquium, pages 1-13, 2001.

[36] M.R. Shapland. Using the odp boostrap model: a practitioners guide. CAS MONOGRAPH SERIES NUMBER 4, 2016.

[37] CAS Working Party. The analysis and estimation of loss and alae variability: a summary report. CAS Forum, Fall: 29-146, 2005. 


\section{Appendix}
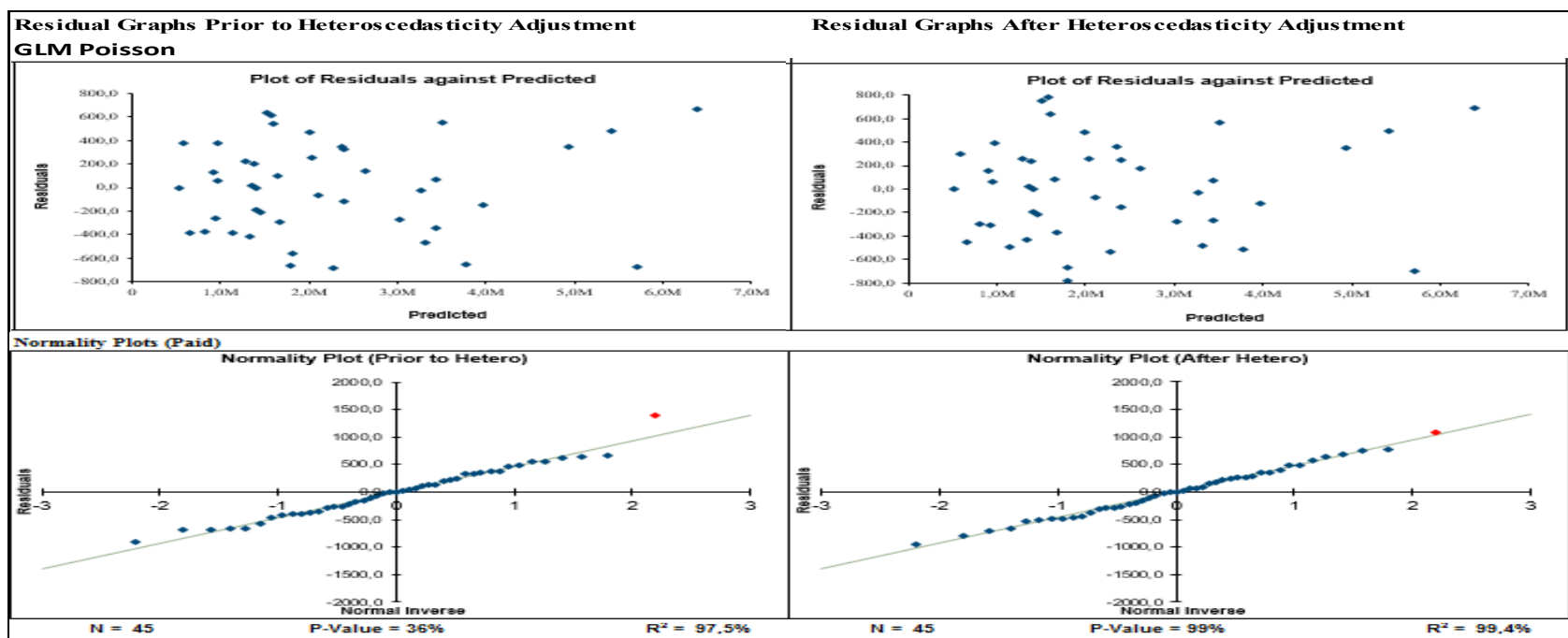

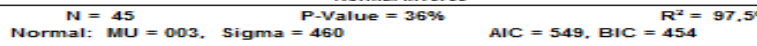
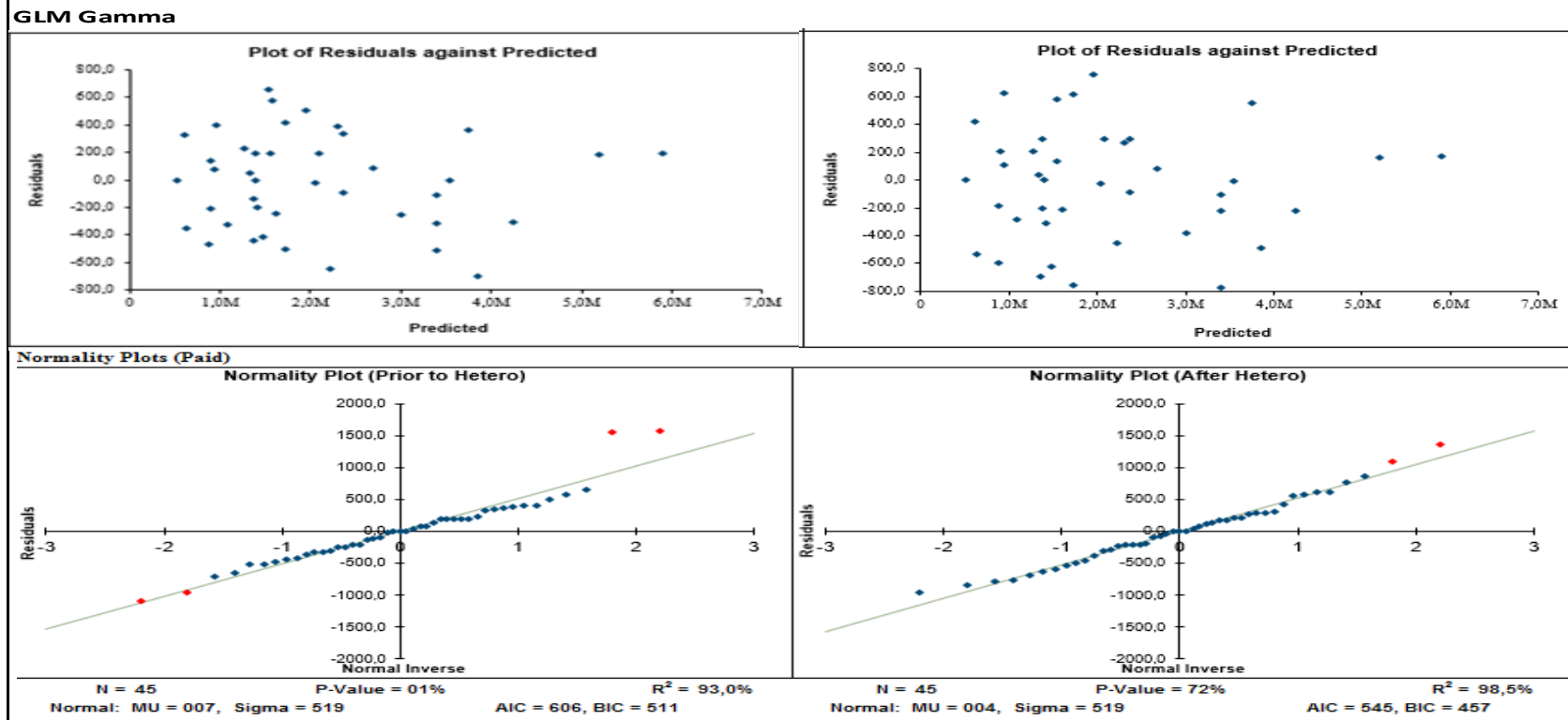

GLM Normal
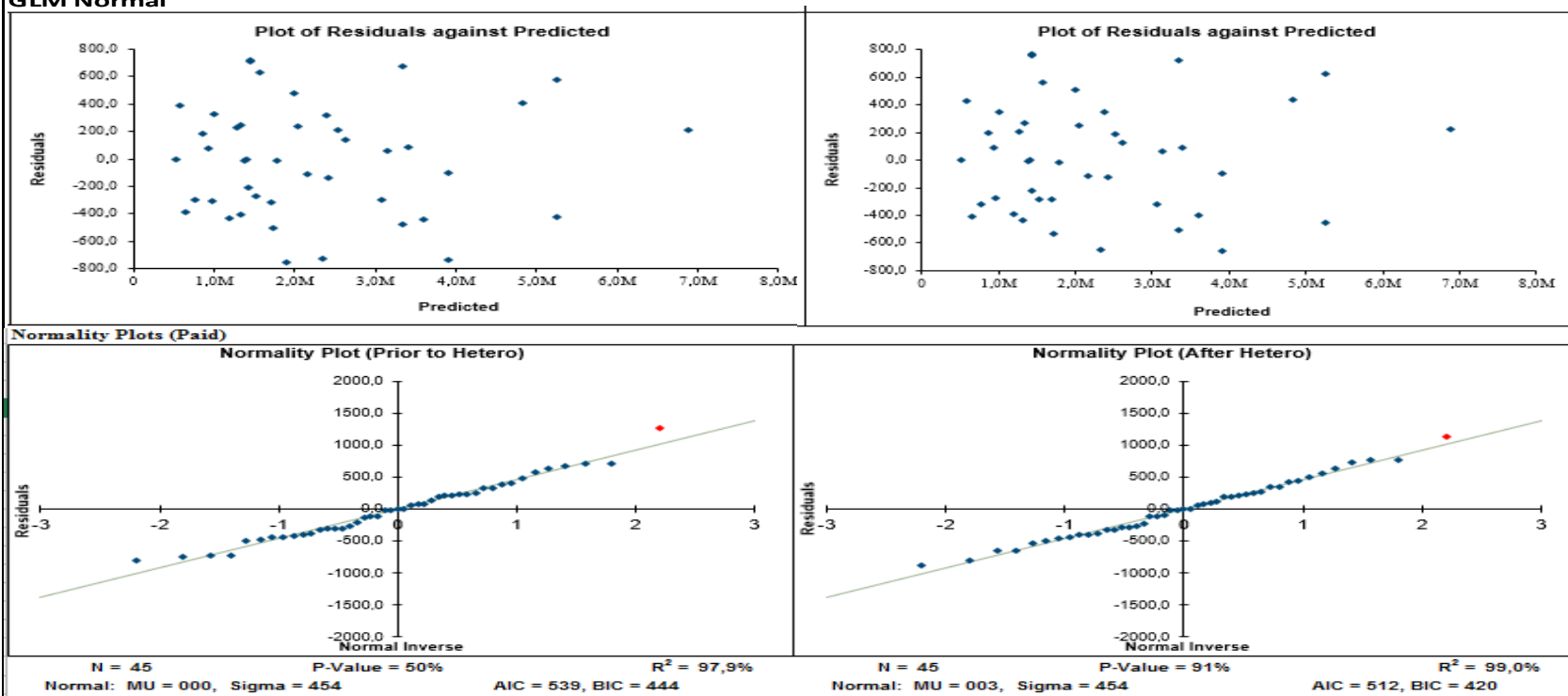

Normal: $M U=000$, Sigma $=454$

AIC $=539$, BIC $=\begin{array}{r}R^{2} \\ 444\end{array}$

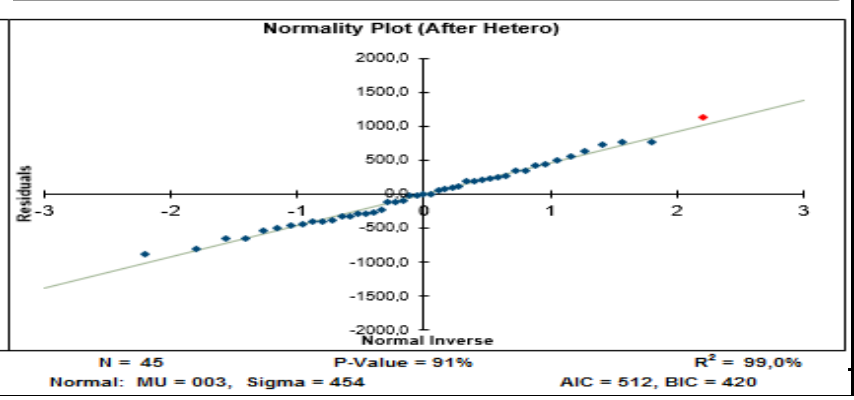


A1.1: Diagnostic tests of residuals (Data 1 - Full Model)

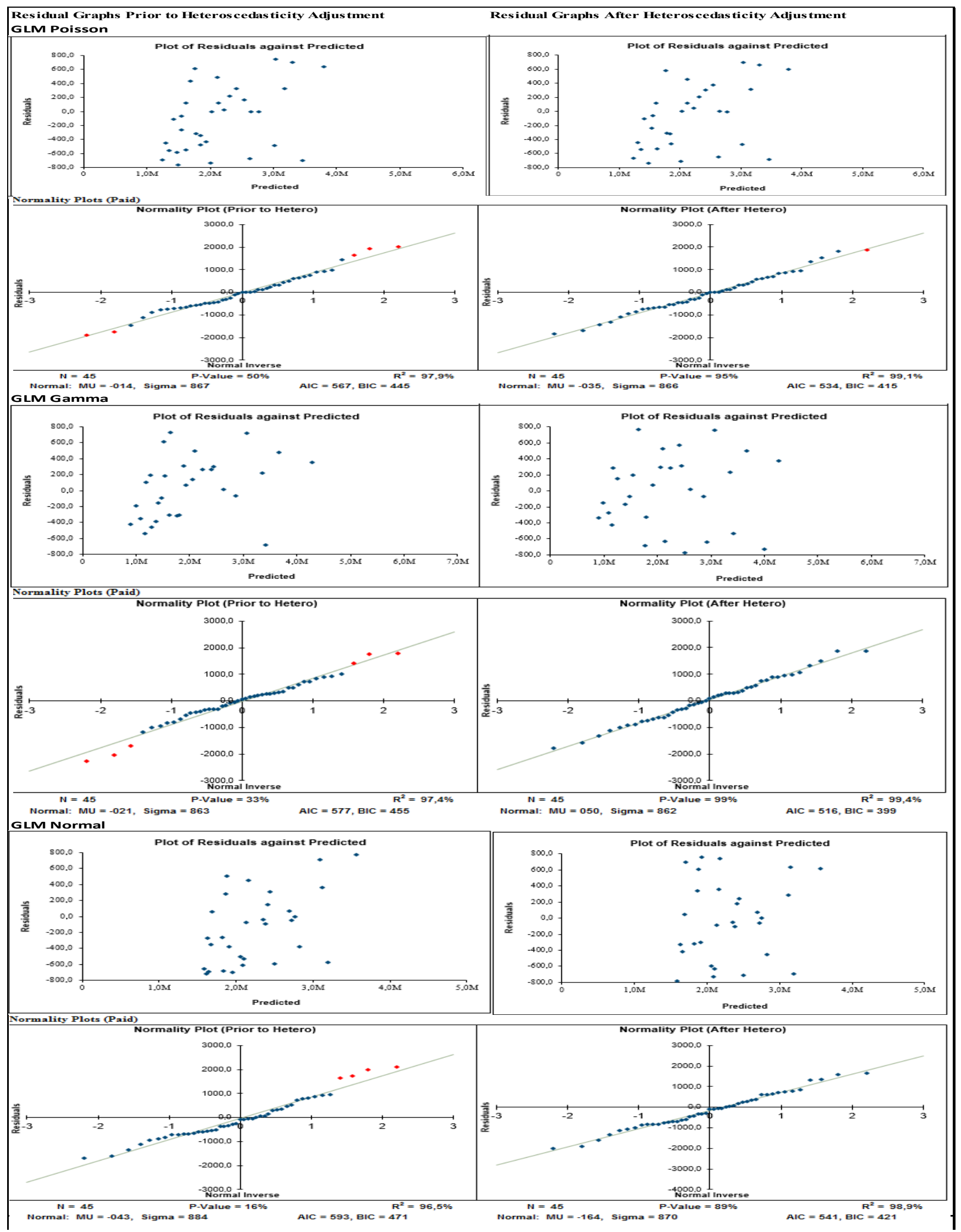

A1.2: Diagnostic tests of residuals (Data 1 - Restricted Model) 

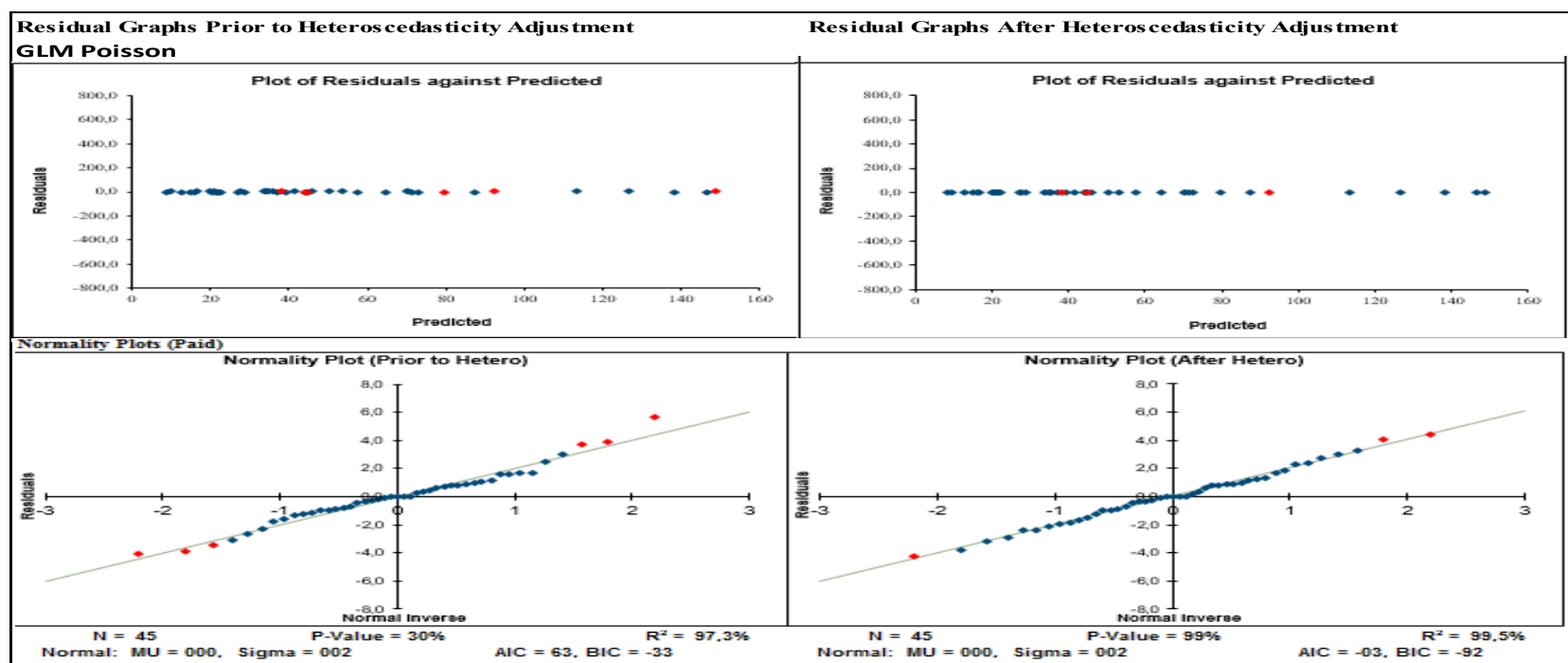

GLM Gamma
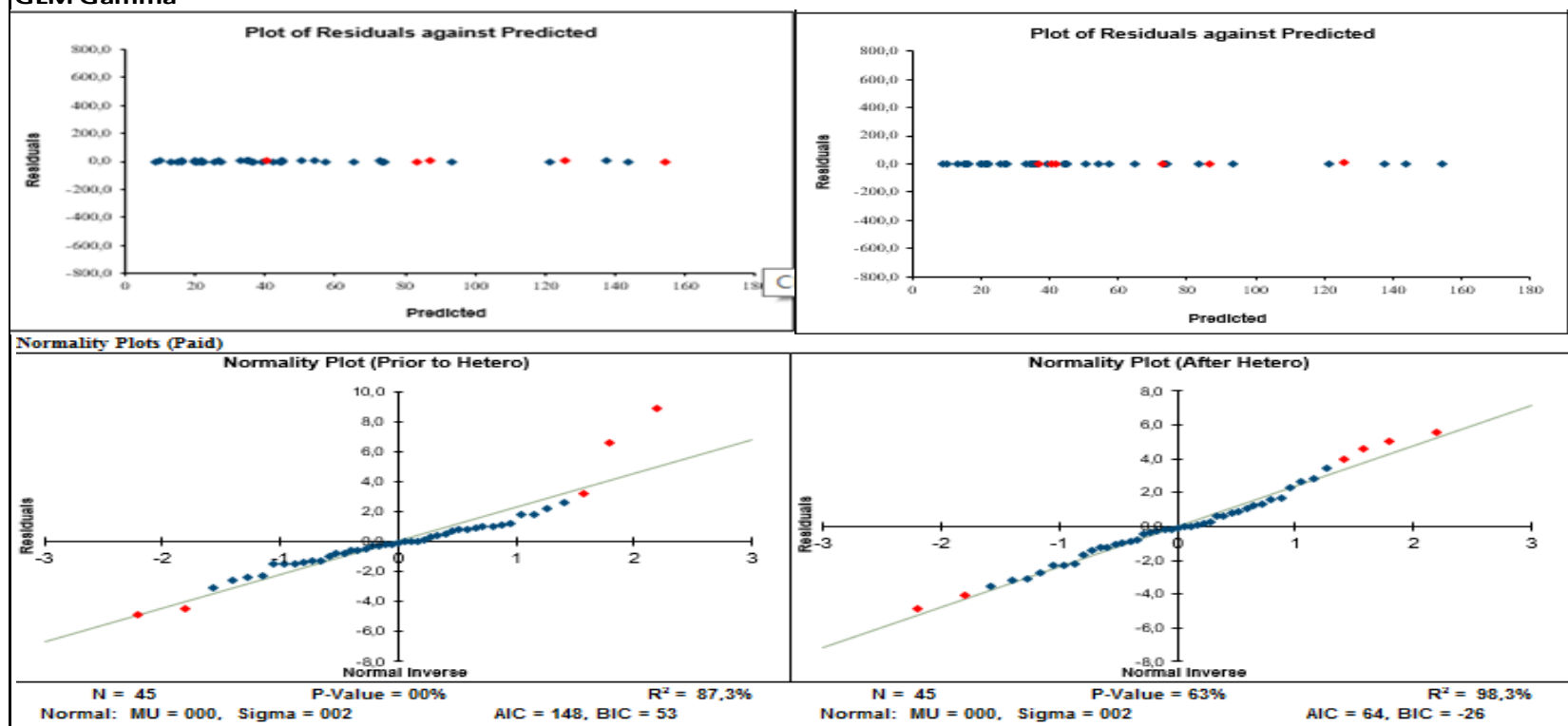

GLM Normal
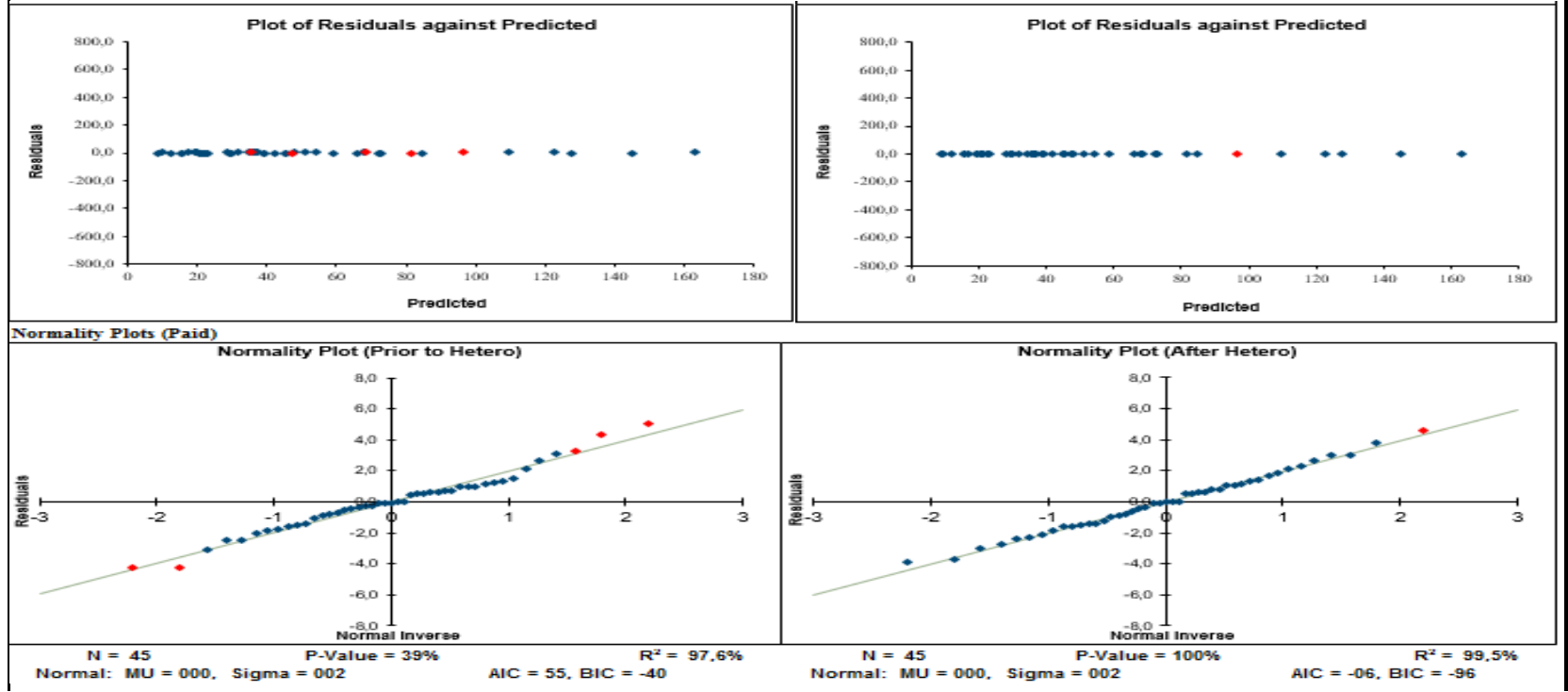

A2.1: Diagnostic tests of residuals (Data 2 - Full Model) 


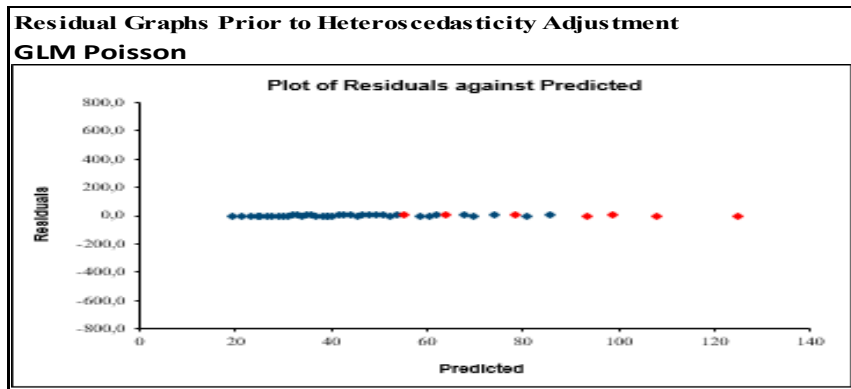

Residual Graphs After Heteros cedas ticity Adjus tment
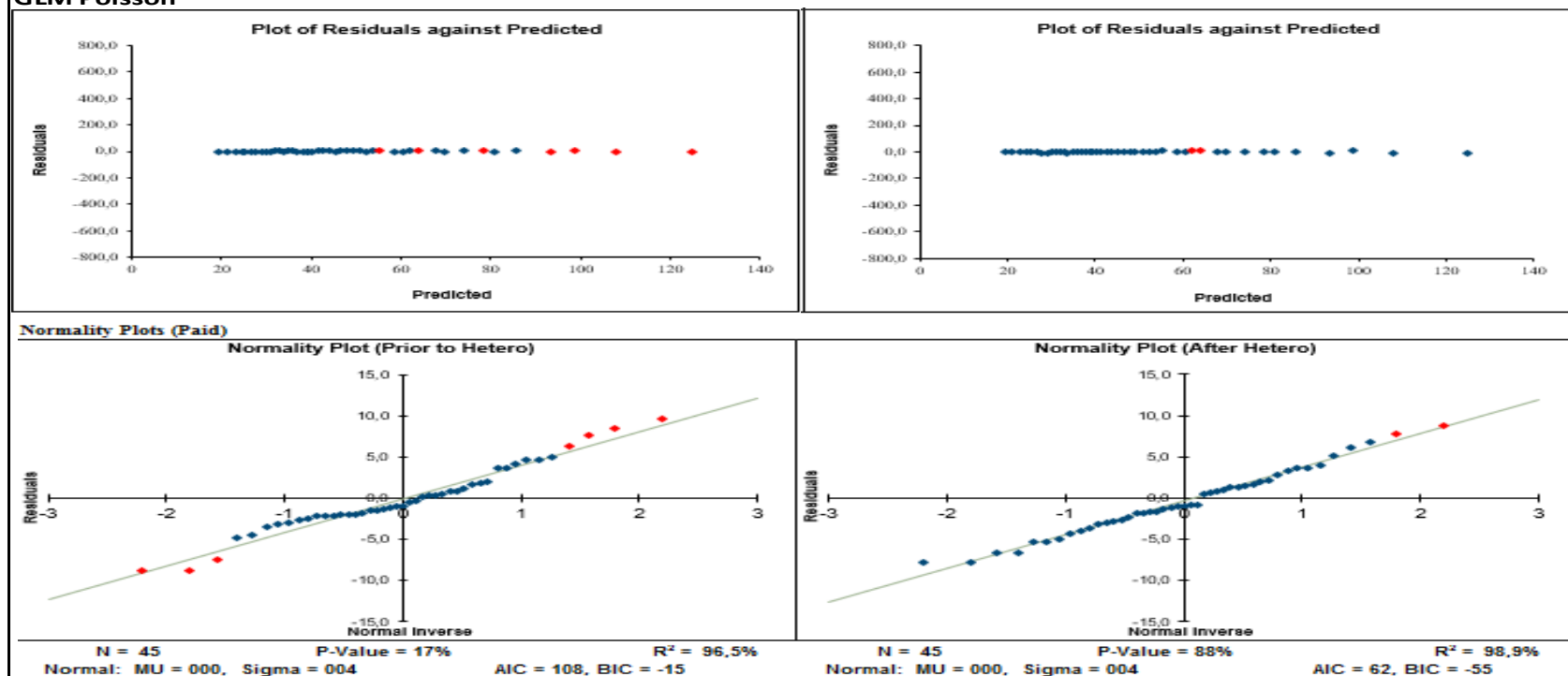

GLM Gamma
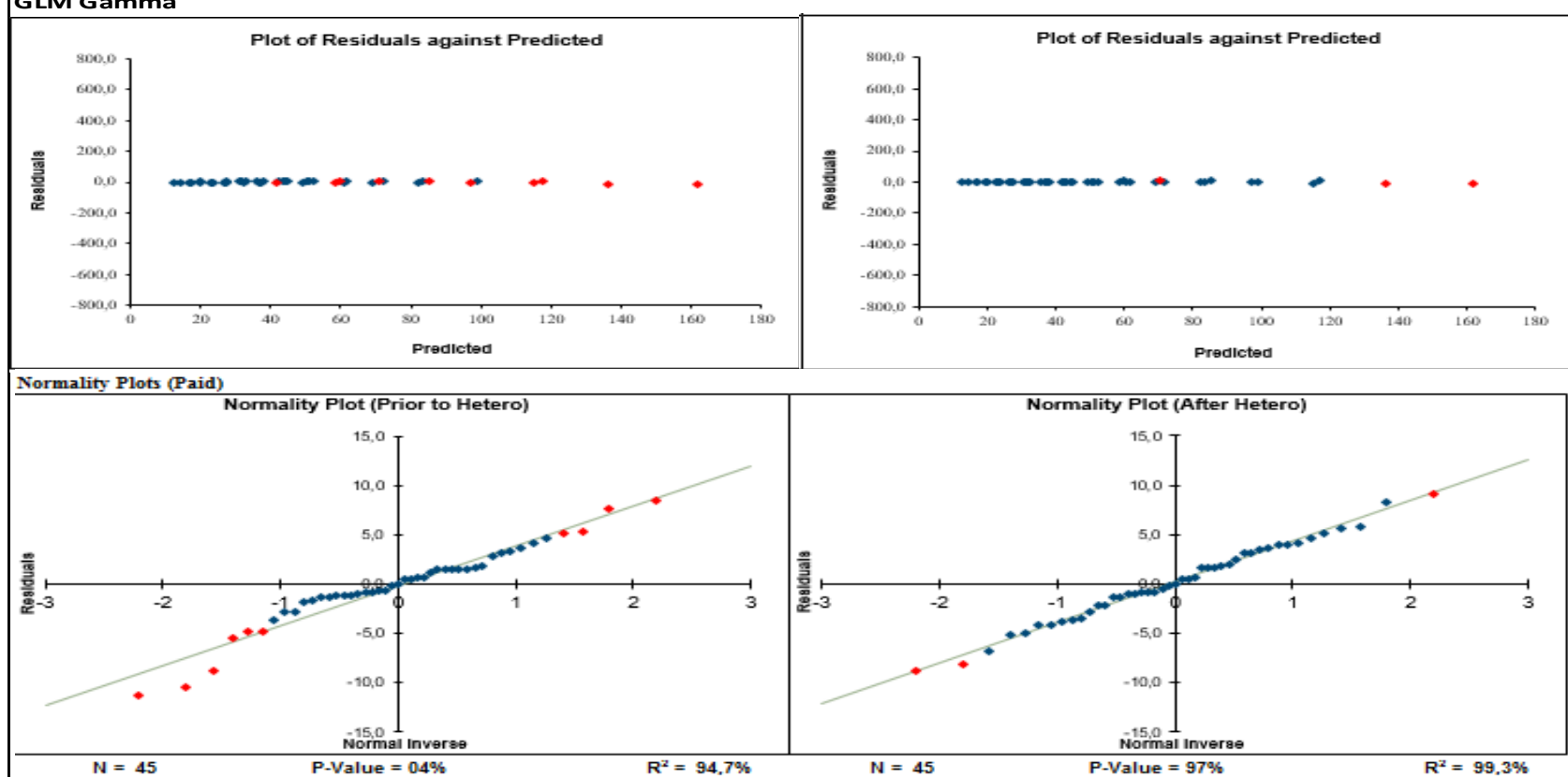

$\begin{array}{ccc}N=45 & \text { P-Value }=04 \% & \text { AIC }=127, \text { BIC }=04 \\ \text { Normal: } M U=000, & \text { Sigma }=004\end{array}$ GLM Normal
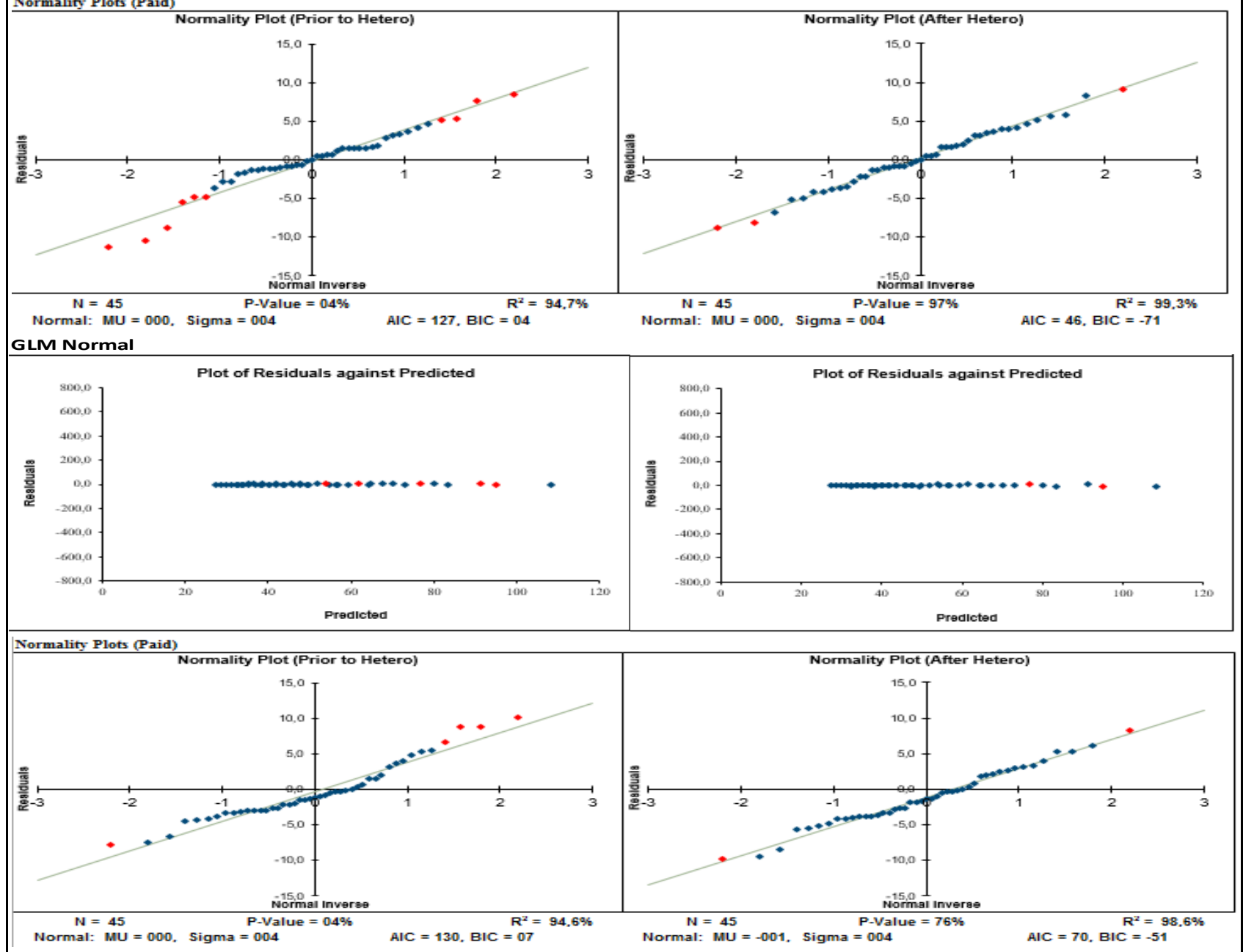

A2.2: Diagnostic tests of residuals (Data 2 - Restricted Model) 
Residual Graphs Prior to Heteroscedas ticity Adjustment GLM Poisson

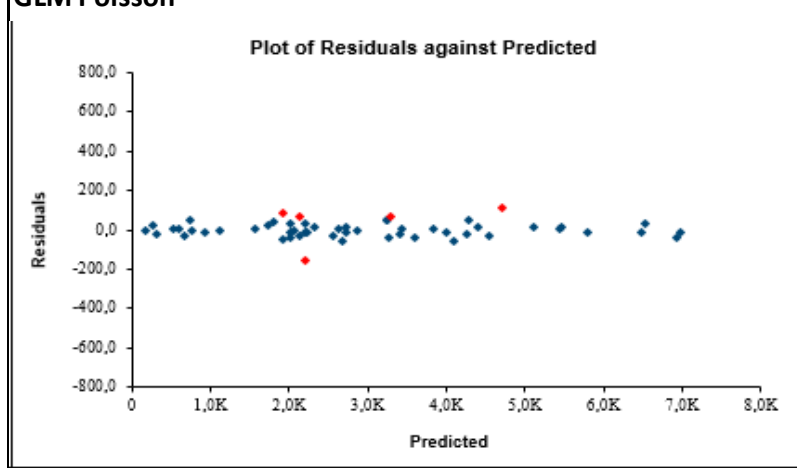

Normality Plots (Paid)

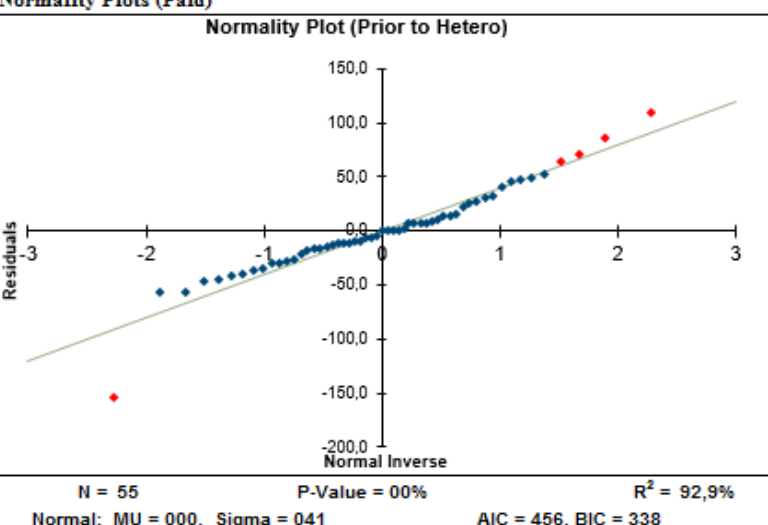

Normal: $M U=000$, Sigma $=041$

GLM Normal
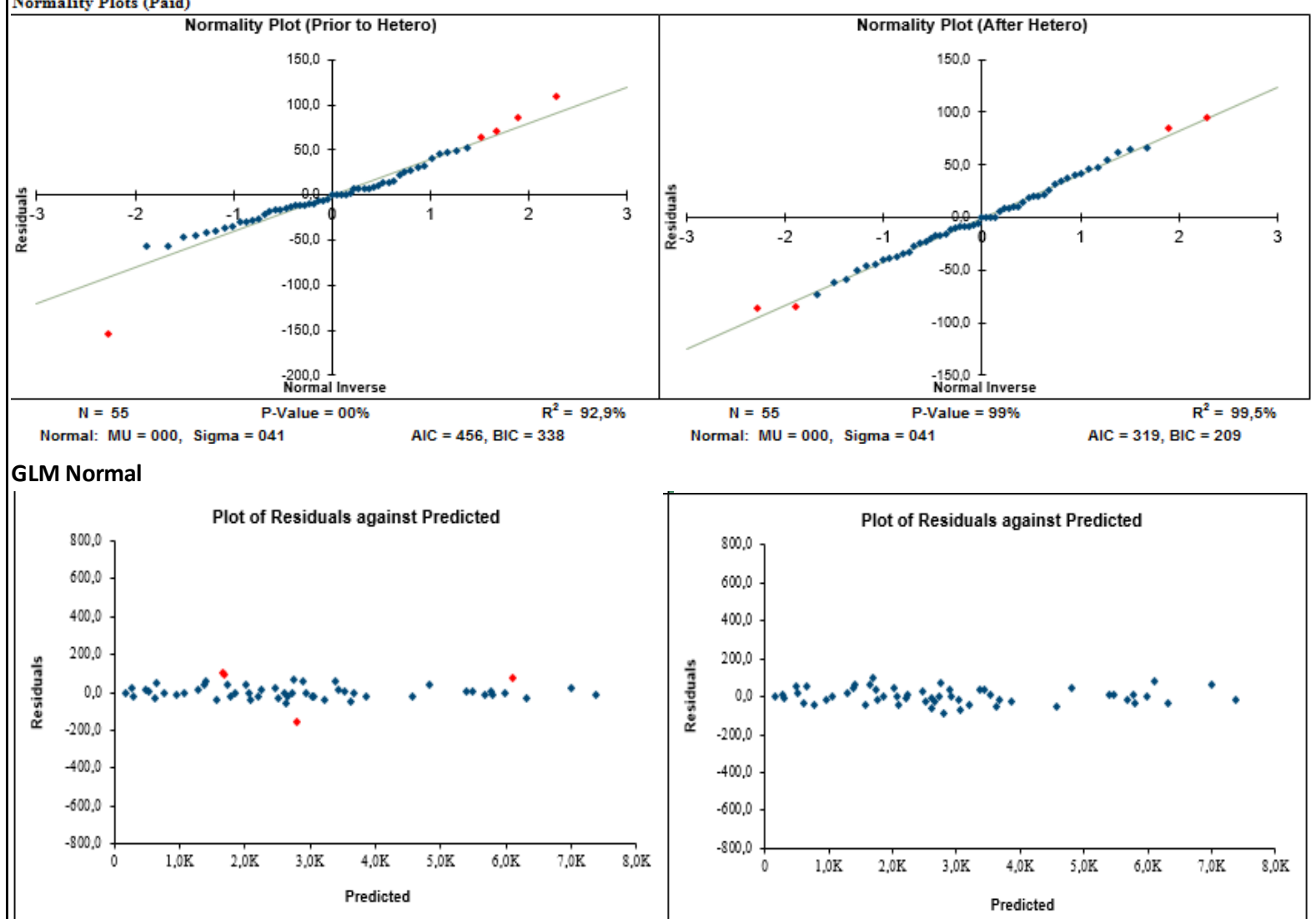

Normal: $M U=000$, Sigma $=041$

AIC $=319, B I C=209$

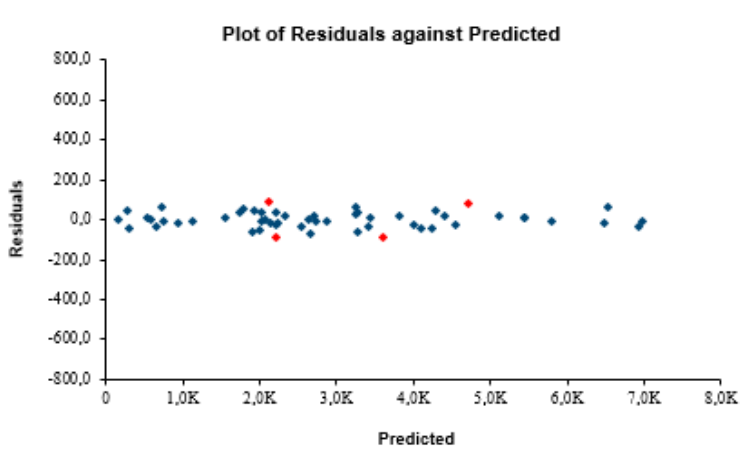

Residual Graphs After Heteros cedasticity Adjus tment

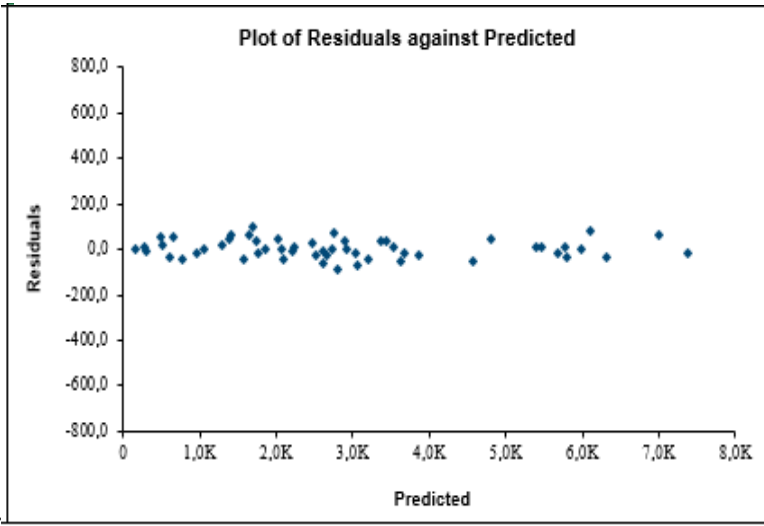

Normality Plots (Paid)

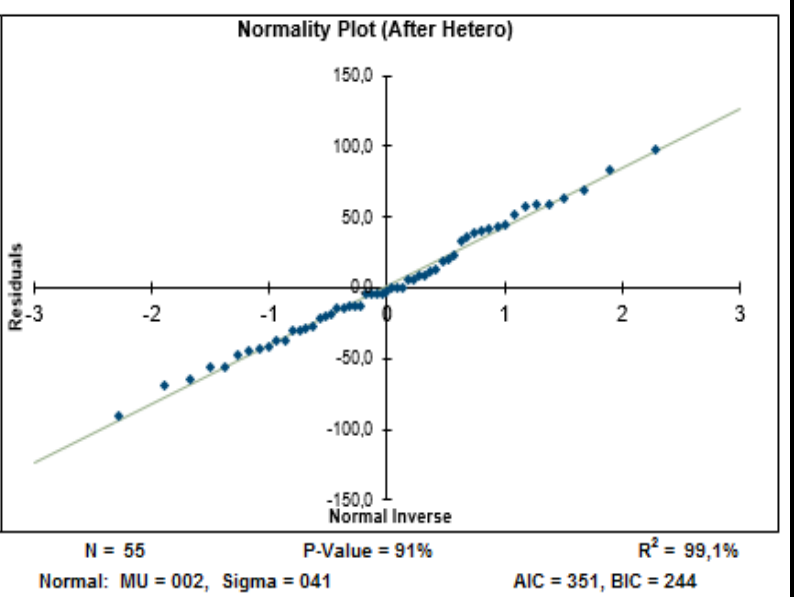

Normal: $\mathrm{MU}=002$, Sigma $=041$ Normal: $M U=002$, Sigma $=041$ $\mathrm{AIC}=351, \mathrm{BIC}=244$

\section{A3.1: Diagnostic tests of residuals (Data 3 - Full Model)}




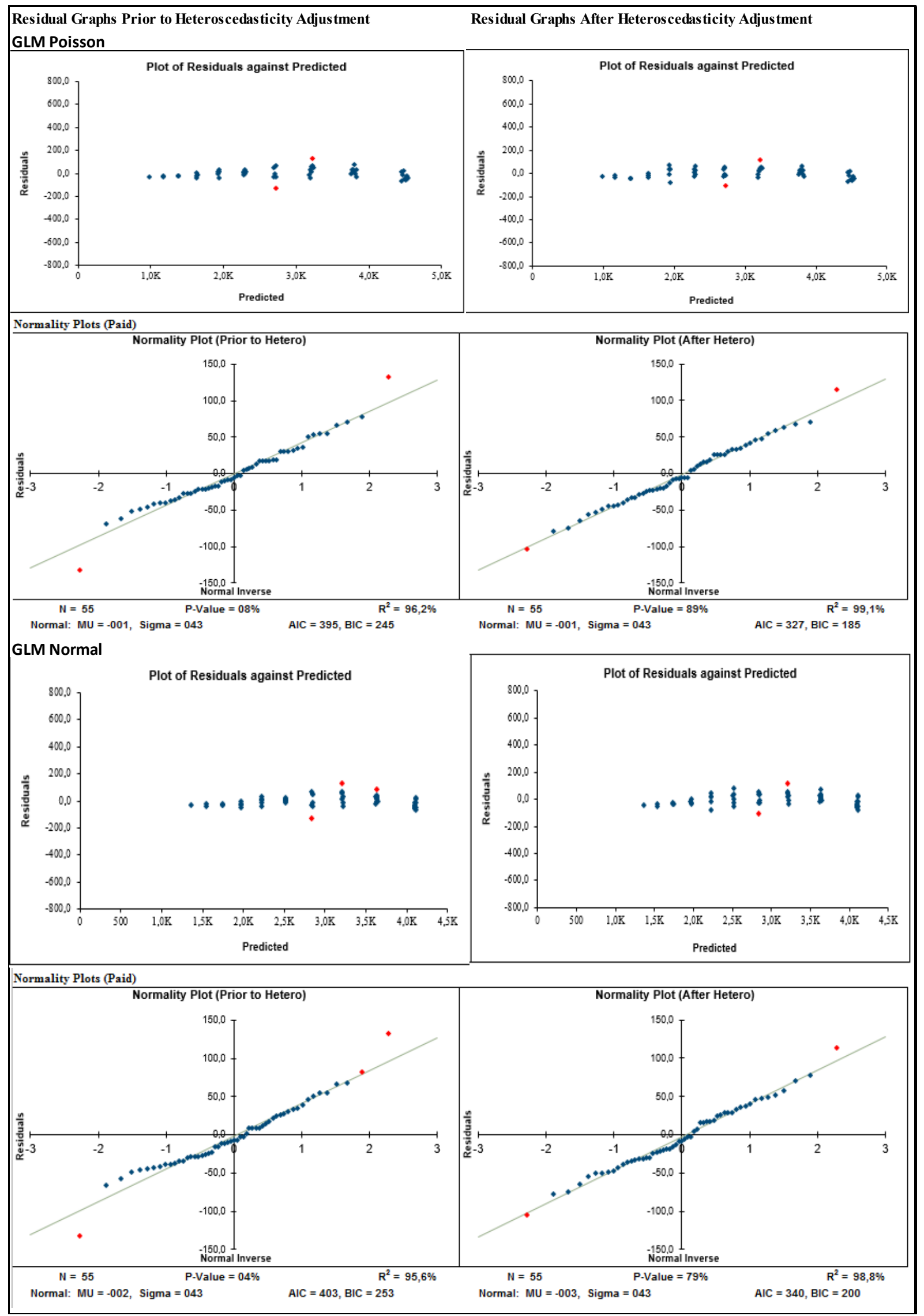

A3.2: Diagnostic tests of residuals (Data 3 - Restricted Model) 